\title{
Current perspectives on hyperthermic intraperitoneal chemotherapy in gastric cancer
}

This article was published in the following Dove Press journal:

Gastrointestinal Cancer:Targets and Therapy

I September 2017

Number of times this article has been viewed

\section{Sean D Smith \\ Daniel Kirkpatrick \\ Peter Thomas \\ Brian W Loggie}

Department of Surgery, Creighton University School of Medicine,

Omaha, NE, USA
Correspondence: Peter Thomas Department of Surgery, Creighton University, 2500 California Plaza, Criss III, Rm 254, Omaha, NE 68I78, USA

Tel + I 402280392 I

Fax + I 402280346 I

Email peterthomas@creighton.edu
Abstract: Cytoreductive surgery with hyperthermic intraperitoneal chemotherapy (CRSHIPEC) is an effective treatment in a variety of cancers with peritoneal carcinomatosis (PC), especially in pseudomyxoma peritonei for which it is the standard of care. The data for gastric cancer are not as conclusive. This review discusses the effect of HIPEC on gastric cancer survival, the importance of patient selection, the effect of HIPEC on gastric cancer morbidity, translational research on HIPEC, and other recent research on HIPEC in the setting of gastric cancer and/or peritoneal metastases. CRS-HIPEC has been shown to be superior to either CRS alone or systemic chemotherapy alone in terms of improving survival. CRS-HIPEC seems to be the best current treatment for patients with gastric cancer and peritoneal metastases. The international community has recommended it as the treatment for this disease. Prudent patient selection before CRS-HIPEC is recommended as subgroups of patients have been shown to benefit from the treatment, while others have not. Studies on CRS-HIPEC have shown the procedure to have acceptably low rates of morbidity and peri- and postoperative complications as well as significant reductions in the incidence of ascites associated with PC. Translational research on HIPEC supports its use as prophylaxis for prevention of peritoneal metastasis and demonstrates HIPEC to be both effective and safe. Measurement of the tumor marker carcinoembryonic antigen has been shown to be an effective indicator of future outcomes in gastric cancer treated with CRS-HIPEC. Although the treatment outcomes have improved, even current treatment using CRS-HIPEC for gastric cancer with PC the survival rates can be dismal. Thus, the treatment of advanced gastric cancer with PC is an ongoing field of study and future directions of the treatment of gastric cancer with PC may include the use of intraperitoneal immunotherapy. Keywords: gastric cancer, cytoreductive surgery, HIPEC, morbidity, mortality

\section{Introduction}

Gastric cancer is the third most common cancer in the world with an estimated 950,000 new cases yearly. ${ }^{1}$ While the highest rates of gastric cancer occur in Korea, Mongolia, and Japan, ${ }^{1}$ the American Cancer Society estimates that there will be 28,000 new cases of gastric cancer in the USA during $2017 .{ }^{2}$ While it only represents about $1.5 \%$ of cancers in the USA, it is anticipated that gastric cancer will result in 11,000 deaths or $2.8 \%$ of all US cancer deaths. ${ }^{2}$ Gastric cancer is more common in males by a ratio of $1.65: 1$ and it kills males at a significantly higher rate than females $(4.3: 1) .{ }^{3}$ Gastric cancer metastasizes aggressively, with the most common sites of gastric cancer metastases being: liver (48\%), peritoneum (32\%), lung (15\%), and bone (12\%). ${ }^{3}$ Metastatic spread to the peritoneum carries a particularly poor prognosis: 5 -year survival is $\sim 4 \% .{ }^{4}$ Comparatively, gastric cancer without peritoneal metastases has an estimated 5-year survival rate of 
$29 \% .{ }^{4}$ Untreated, the median survival for patients suffering from gastric cancer with peritoneal dissemination is about 6 months in patients 44 years old or younger and 3 months in patients $>75 .{ }^{5}$ However, recent developments in treatment that include pairing cytoreductive surgery (CRS) and hyperthermic intraperitoneal chemotherapy (HIPEC) have improved survival for some patients with peritoneal metastases to 5 years or more. ${ }^{6}$ HIPEC works via a pharmacokinetic advantage; chemotherapy infused directly into the peritoneum is largely unabsorbed through the peritoneal-plasma membrane and remains at a high concentration in the abdomen with relatively low concentrations in the peripheral blood. As a result, HIPEC increases the direct cytotoxic effect of chemotherapy on peritoneal tumors while simultaneously limiting the systemic adverse effects. ${ }^{7}$ The rationale for hyperthermia in peritoneal infusion is 3-fold: First, hyperthermia causes microvessel embolization and induces ischemic necrosis in tumor tissue; Second, hyperthermia directly kills cancer cells in the $\mathrm{S}$ and $M$ phase of the cell cycle by disturbing cell homeostasis and energy metabolism, activating lysosomes, and destroying the cytoplasm and nucleus. ${ }^{7}$ Third, hyperthermia disrupts cell membrane proteins and interferes with synthesis of DNA, RNA, and proteins. ${ }^{7}$ Complete CRS and HIPEC is now the standard of care for pseudomyxoma peritonei (PMP) and malignant peritoneal mesothelioma (MPM), and it is rapidly becoming the standard of care for carcinomatosis from colorectal cancers. However, the data for gastric cancer are not as conclusive and more studies need to be carried out before CRS and HIPEC are accepted as a standard treatment for gastric cancer. ${ }^{8}$ This review will concentrate on the current treatments for peritoneal metastasis from gastric cancer and will only discuss other sites of metastasis if they impact peritoneal disease. Specifically, this review will discuss: the effect of HIPEC on gastric cancer survival, the importance of patient selection, CRS-HIPEC and the standard of care, the effect of HIPEC on gastric cancer morbidity, translational research on HIPEC, and other recent research on HIPEC in the setting of gastric cancer and/or peritoneal metastases.

\section{The effect of HIPEC on gastric cancer mortality}

There is a significant literature concerning the effect of HIPEC and CRS (CRS-HIPEC) on patients with gastric cancer. These studies have largely shown CRS-HIPEC to increase the overall survival of gastric cancer patients. For example, in a meta-analysis, Ni et al, ${ }^{9}$ showed that the 1-, 3-, and 5-year survival of patients with gastric cancer receiving CRS-HIPEC were significantly longer than patients who received surgery alone. In patients with both HIPEC and surgery, the rate of liver, lung, and bone metastases, as well as peritoneal metastases recurrence, was also lower. ${ }^{9} \mathrm{~A}$ large meta-analysis by Zeng et al, ${ }^{10}$ also showed that treatment with HIPEC was an independent prognostic factor that increased survival times in patients with gastric cancers. A study by Ji et al combined the published clinical trials on CRS-HIPEC. They showed that, untreated gastric cancer with peritoneal metastases lead to death in $<5$ months, CRS increased survival to 7.9 months and CRS-HIPEC increased overall survival to 13.3 months (in cohort studies). ${ }^{11}$ The literature, therefore, supports CRS-HIPEC as a therapy for increasing survival in patients with gastric cancer with peritoneal metastases. The results of selected studies that examined CRS-HIPEC in the setting of gastric cancer with peritoneal metastases are provided in Table 1 with weighted averages and improvements in patient survival by year shown in Tables 2 and 3, respectively.

CRS-HIPEC has been compared with CRS alone and shown to improve survival. In a randomized controlled trial conducted by Yang et al, ${ }^{12}$ patients treated with CRS-HIPEC had significantly increased median survival times over patients treated with CRS alone (11 vs 6.5 months, respectively) with no difference in postoperative morbidity. In a large meta-analysis of 20 prospective randomized controlled trials between 1987 and 2011, Coccolini et al, ${ }^{13}$ compared surgery with intraperitoneal chemotherapy (IPC), the majority of which was HIPEC, with radical resection without IPC. Results showed increased 1-, 2-, and 3-year mortality rates, decreased rates of peritoneal recurrence, and decreased rates of hematogenous metastasis in the group analogous to CRS-HIPEC. ${ }^{13}$ Likewise, Boerner et al ${ }^{14}$ showed that the combination of HIPEC with CRS doubled patients' 2-year survival rates (35.8\% vs $16.9 \%)$ compared with traditional chemotherapy with gastrectomy. Impressively, this study showed that patients treated with CRS-HIPEC have comparable survival to matched patients who do not have peritoneal metastases. ${ }^{14} \mathrm{Wu}$ et $\mathrm{al}^{15}$ studied patients with gastric cancer who had ovarian and peritoneal metastases and compared CRS alone with CRS-HIPEC. They showed improved median survival times in the HIPEC group in those with peritoneal metastases, but no difference between groups in those with ovarian metastases alone, they therefore recommend using CRS-HIPEC for gastric cancer with peritoneal metastases, but do not recommend it for the use of ovarian metastases alone. $^{15}$

HIPEC has also been compared with systemic chemotherapy for peritoneal metastasis and showed increased rates 
Table I Results of selected studies on patients with advanced gastric cancer treated with CRS-HIPEC

\begin{tabular}{|c|c|c|c|c|c|c|c|}
\hline Author & Publication year & Patients & I year $(\%)$ & 2 years $(\%)$ & 3 years $(\%)$ & 5 years $(\%)$ & Median (months) \\
\hline Hall et $\mathrm{al}^{35}$ & 2004 & 34 & 45 & 16 & - & - & 11.2 \\
\hline Glehen et $\mathrm{a}^{20}$ & 2010 & 159 & 43 & 18 & 13 & - & 9.2 \\
\hline Yang et $\mathrm{al}^{42}$ & 2010 & 28 & 50 & 43 & - & - & 27.7 \\
\hline Li et $\mathrm{al}^{27}$ & 2010 & 128 & 52.5 & - & 13.2 & 5.5 & 11.8 \\
\hline Yang et $\mathrm{al}^{\prime 2}$ & 2011 & 68 & - & - & - & - & 11 \\
\hline Wu et $\mathrm{al}^{50}$ & 2013 & 62 & - & - & - & - & 15.5 \\
\hline Magge et $\mathrm{a}^{37}$ & 2014 & 23 & 50 & - & 18 & - & 9.5 \\
\hline Canbay et $\mathrm{a}^{23}$ & 2014 & 194 & 66 & 32 & - & 10.7 & 15.8 \\
\hline Yarema et $\mathrm{a}^{25}$ & 2014 & 49 & 68.8 & - & - & - & 12 \\
\hline Saladino et $\mathrm{al}^{24}$ & 2014 & 12 & - & - & - & - & 24 \\
\hline Müller et $\mathrm{a}^{43}$ & 2014 & 26 & - & 38 & - & - & 19 \\
\hline Wu et $a^{160}$ & 2015 & 26 & - & - & - & - & 28.2 \\
\hline Desantis et $\mathrm{al}^{38}$ & 2015 & 14 & - & - & - & - & 8.1 \\
\hline $\mathrm{Tu}^{36}$ & 2016 & 231 & 83.4 & 68.5 & 38.7 & - & 37 \\
\hline Geng et $\mathrm{a}^{28}$ & 2016 & 312 & 66.7 & 28.5 & - & - & 17 \\
\hline Yuan et al ${ }^{16}$ & 2016 & 23 & 41.7 & - & - & - & 16.5 \\
\hline Boerner et $\mathrm{al}^{14}$ & 2016 & 38 & - & 35.8 & - & - & 17.2 \\
\hline Wu et $\mathrm{al}^{39}$ & 2016 & 30 & 77.5 & 32.5 & 19.8 & - & 24.2 \\
\hline Chia et al ${ }^{56}$ & 2016 & 81 & - & - & - & 18 & 17.3 \\
\hline
\end{tabular}

Notes: Compilation of the results of various reviewed studies in terms of survival (median and by year). '-' indicates data not included.

Abbreviations: CRS, cytoreductive surgery; HIPEC, hyperthermic intraperitoneal chemotherapy.

Table 2 Weighted averages of patient survival of selected studies using CRS-HIPEC treatment for advanced gastric cancer

\begin{tabular}{ll}
\hline Average patient survival & $\% /$ months \\
\hline Weighted average I year CRS-HIPEC survival: & $63.73 \%$ \\
Weighted average 2 year CRS-HIPEC survival: & $36.94 \%$ \\
Weighted average 3 year CRS-HIPEC survival: & $24.00 \%$ \\
Weighted average 5 year CRS-HIPEC survival: & $11.16 \%$ \\
Median weighted average CRS-HIPEC survival: & 18.43 months \\
\hline
\end{tabular}

Abbreviations: CRS, cytoreductive surgery; HIPEC, hyperthermic intraperitoneal chemotherapy.

Table 3 Weighted average of median survival of patients treated with CRS-HIPEC for advanced gastric cancer by year

\begin{tabular}{ll}
\hline Average median survival by year & Months \\
\hline 2004 & 11.2 months \\
2010 & 11.9 months \\
2011 & 11.0 months \\
2013 & 15.5 months \\
2014 & 15.3 months \\
2015 & 21.2 months \\
2016 & 23.7 months \\
\hline
\end{tabular}

Abbreviations: CRS, cytoreductive surgery; HIPEC, hyperthermic intraperitoneal chemotherapy.

of survival. ${ }^{16-18}$ A retrospective study by Yuan et al, ${ }^{16}$ showed a 1 -year survival rate of $41.7 \%$ in patients receiving HIPEC and a corresponding 1-year survival of $23.8 \%$ for the systemic chemotherapy group. Rudloff et al, ${ }^{17}$ also did a retrospective study on patients treated with multimodal therapy (CRS, HIPEC, and systemic chemotherapy) vs patients treated with systemic chemotherapy alone and showed the median overall survival to be 11.3 months in the multimodal treatment group compared with 4.3 months in the traditional chemotherapy group. Notably, no patients in the chemotherapy-alone group lived beyond 11 months, while one patient in the multimodal treatment group was still living 4 years after treatment. ${ }^{17}$ Zhibing et al, ${ }^{18}$ retrospectively reviewed 101 patients with advanced gastric cancer and separated them into 2 groups: 49 patients receiving traditional intravenous chemotherapy and 52 patients receiving HIPEC. They found an increased response rate and efficacy in the HIPEC treatment group, as well as a significantly increased disease-free survival time. They also reported no statistically significant difference in the side effects: both groups tolerated treatment well. ${ }^{18}$

Combinations of HIPEC and systemic chemotherapy have been a topic of interest in recent research, with some studies exploring the synergistic effects of the 2 modes of treatment. Yonemura et al, ${ }^{19}$ studied 105 gastric cancer patients with peritoneal metastases and showed that neoadjuvant laparoscopic HIPEC (NLHIPEC) with systemic chemotherapy improved the patient's' prognosis. They measured these outcomes with the peritoneal cancer index (PCI). Previous studies have shown that a PCI $>12$ was associated with shorter term survival from gastric cancer even after CRS. ${ }^{20}$ Adding NLHIPEC and systemic chemotherapy significantly improved the PCI, qualifying patients for CRS, and increased the median survival time of gastric cancer patients from 8.6 to 19.2 months. Schildberg et $\mathrm{al}^{21}$ conducted a similar study in which 76 patients with metastatic gastric cancer who were initially identified as "unresectable" and subsequently underwent palliative chemotherapy were re-evaluated as resectable 
and underwent CRS-HIPEC. This group showed significant survival advantages compared with palliative care. Some of the patients who had been deemed unresectable and ultimately received CRS-HIPEC even achieved long-term survival. ${ }^{21}$ Similarly, Wu et $\mathrm{al}^{22}$ examined the survival benefit of CRS complete resection with the use of postoperative adjuvant systemic chemotherapy and HIPEC. They found that combining these treatments after CRS improved overall survival and decreased the recurrence rates at 1,3 and 5 years with a low rate of chemotherapy-associated toxicities $(<10 \%))^{22}$ An interesting new approach to HIPEC was studied by Canbay et al. ${ }^{23}$ This group used bidirectional intraperitoneal and systemic induction chemotherapy, which combines HIPEC simultaneously with systemic chemotherapy, followed by CRS-HIPEC. ${ }^{23}$ They found increased survival rates over CRS-HIPEC alone, especially in patients with sensitivity to the chemotherapy. ${ }^{23}$ Many different applications of HIPEC, systemic chemotherapy, and CRS have been studied. Despite this body of research, and many trials showing positive results, no standard has been set for which treatment modality should be preferred.

Multiple studies have shown that in patients with a high risk for peritoneal metastasis, adjuvant or prophylactic treatment with HIPEC improves outcomes. ${ }^{24-26}$ Macri et $\mathrm{al}^{46}$ reported a very low rate of peritoneal occurrences $(8.3 \%)$ with prophylactic HIPEC compared with no treatment (50\%) in high-risk patients. They attribute this impressive reduction in peritoneal metastases to HIPEC's ability to damage cancer cells or micrometastases through the synergistic combination of heat and chemotherapy. ${ }^{24}$ Yarema et al, ${ }^{25}$ similarly report that adjuvant HIPEC in gastric cancer patients with a high risk for peritoneal carcinomatosis (PC) had a PC rate of $11.1 \%$ compared with $73.7 \%$ in a control group. ${ }^{25}$ This reduction in PC led to a significantly improved patient survival rate. ${ }^{25}$ HIPEC, thus, holds promise as a prophylactic treatment for gastric cancer patients with a high risk of developing PC.

Much of the research evaluates the combination of HIPEC with CRS as a means of treating advanced gastric cancer. In a somewhat different vein, many studies evaluated the benefit of combining HIPEC and gastrectomy in different modalities. An early study by Costa et al, ${ }^{26}$ showed that in a small cohort of 10 patients at high risk for PC from gastric cancer, multimodal treatment with neoadjuvant systemic chemotherapy followed by HIPEC and gastrectomy produced long-term survival in 7 out of the 10 patients. They emphasized that larger studies were needed with similar methodology before significant conclusions can be reached.
Li et al conducted a larger study of 128 patients comparing a gastric resection group with HIPEC to a non-gastrectomy group. They found significantly higher 5-year survival rates, prognosis, and overall survival in the HIPECgastrectomy group. ${ }^{27}$ Similarly, Geng et al, ${ }^{28}$ looked retrospectively at over 300 Chinese patients with advanced gastric cancer and showed a benefit for combining gastrectomy with HIPEC. Patients that received HIPEC and gastrectomy had higher median survival rates and higher 1- and 2-year survival rates when compared with the other groups in the study. Interestingly, in Geng et al's, ${ }^{28}$ study population, when chemotherapy was not given to the gastrectomy group, survival was not statistically changed. In other words, this study seems to show a survival benefit to HIPEC in a setting where traditional chemotherapy does not significantly alter mortality. Work by Graziosi et al, ${ }^{29}$ also showed benefit with gastrectomy combined with HIPEC in a high-risk patient population as a means of prophylaxis. They concluded that prophylactic HIPEC with gastrectomy is feasible and increases survival over surgery alone. ${ }^{29}$ Research by Coccolini et al built on this work by comparing the survival of patients treated with prophylactic HIPEC and gastrectomy to patients treated with gastrectomy alone. They found an association between prophylactic HIPEC and increased disease-free survival (DFS) and overall survival (DFS surgery alone 21.6 vs 34.5 months in prophylactic group). ${ }^{30}$ A study of 112 surgeries by Kang et $\mathrm{al}^{31}$ compared patients treated with gastrectomy alone against patients treated with gastrectomy plus HIPEC with impressive results: 5-year survival rates of $43.9 \%$ in CRS-HIPEC (vs $10.7 \%$ in surgery alone) and DFS rates of $66 \%$ in the CRS-HIPEC group (vs $28.8 \%$ in the surgery-alone group). Importantly, this study also showed no difference in the rate of complications between the 2 groups. ${ }^{31}$

Other studies have emphasized the importance of removing other organs during the cyto-reductive portion of the CRS-HIPEC regimen. One study, for example, recommended including resection of the round ligament of the liver and the gallbladder. ${ }^{32}$ At the same time, other studies have found that CRC-HIPEC has greatest efficacy when fewer organs are involved: extreme multivisceral resection was associated with higher major morbidity and inferior outcomes. ${ }^{33}$ It is important to note that comparing the survival of those with extreme tumor burdens to those with lesser tumor burdens may be biased in its approach and produce deceptive data. Thus, visceral sparing seems to be important for overall survival and quality of life in the gastric cancer patient with peritoneal metastasis. 


\section{Importance of patient selection}

Many articles emphasize the importance of careful patient selection in the use of CRS-HIPEC, showing increased survival among certain groups of patients. An early study of the effectiveness of CRS-HIPEC for use in primary gastric cancer with PC found that resection status after surgery was significantly correlated with improved survival. ${ }^{34,35}$ Specifically, they found that patients who underwent an R0/R1 resection had improved survival compared with patients that obtained an R2 status post-surgery. ${ }^{36}$ The authors emphasized that careful patient selection is imperative, noting that only those who can achieve an R0/R1 status should be candidates for CRS-HIPEC. Selection criteria that have been shown to improve survival rates include fewer organ involvement/low tumor stage ${ }^{23,33,36,37}$; World Health Organization Performance Status, ${ }^{37}$ previous chemotherapy, ${ }^{10,12,37,38}$ completeness of cytoreduction score, ${ }^{2,12,35-41}$ and a low PCI., ${ }^{2,17,20,37,40-44}$ Table 3 shows the median survival rates of patients from selected trials by year. Interestingly, this table seems to demonstrate increasing CRS-HIPEC survival over time, a relationship that may be linked to improvements in patient selection.

While some patient characteristics have been shown to improve the efficacy of CRS-HIPEC, other characteristics have not. These include in vitro chemotherapy sensitivity ${ }^{45}$ and patient age ${ }^{20,46,47}$ For example, a study by Votanopoulos et al, ${ }^{48}$ examined CRS-HIPEC in the elderly (mean age was 73 years old) and concluded that age alone was not a contraindication for the procedure. Votanopoulos et al support stringent patient selection (including some of the above metrics) and noted that using these metrics for patient selection was key for prolonged survival..$^{48}$ Similar conclusions were reached in a study by Macrì et al. ${ }^{46}$ Although some studies have shown patient age to be negligible in CRS-HIPEC patient selection, patients $>60$ years of age with tumor stage $\mathrm{T} 3$ or T4 have been associated with a higher risk of gastric cancer PC. ${ }^{47}$ In other words, in the absence of other known factors that affect response to CRS-HIPEC, patient age should not be a contraindication to receiving CRS-HIPEC.

\section{CRS-HIPEC and the standard of care}

The standard of care for treatment of PMP is CRS-HIPEC..$^{9}$ PMP has been shown to have the best response of any cancer to this treatment modality and boasts 5-year survival rates of $73 \% .{ }^{49}$ Many studies compared different types of primary cancer, including ovarian cancer, colon cancer, appendiceal tumors, etc. with encouraging results. Desantis et al performed 401 CRS-HIPEC procedures on 356 patients with PC from various primary cancers. They found median survivals of 47.6 months for PC of ovarian cancer origin, 45.8 months in colorectal origin and 64.2 months for PC of peritoneal mesothelioma origin. ${ }^{37}$ Similarly, Votanopoulos et al studied CRS-HIPEC outcomes in various primary cancers, all of which had PC, in patients $>70$ years of age. Their results showed similar encouraging numbers for various forms of PC: median survivals of 31.8 months for appendiceal cancer, 41.5 months for mesothelioma, 54 months for ovarian cancer and 13.2 months for colon cancer. ${ }^{48} \mathrm{Wu}$ et al evaluated the combination of HIPEC and CRS in women with Krukenberg ovarian tumors that had metastasized to the peritoneum. Their analysis shows that HIPEC with surgery improves survival by 5 months when compared with CRS alone. ${ }^{49}$ Importantly, this research also showed that surgery with HIPEC did not have a significant difference in complication rates when compared with surgery alone. ${ }^{49}$ Given the results of studies like these, CRS-HIPEC has become the recommended standard treatment for a variety of cancers with PC, including PMP, MPM, appendiceal mucinous cancer, colorectal PC, and ovarian cancer.

While there is a significant body of evidence supporting the use of HIPEC with surgery to improve the mortality of patients with advanced gastric cancer, some authors have noted that larger studies with more data are needed to definitively establish these techniques as the standard of care. ${ }^{26,29,51,52}$ Regardless, the evidence is strong enough to support CRS with HIPEC as the recommended treatment for gastric cancer with peritoneal metastases. ${ }^{7}$ In fact, in 2016 China defined this therapy as the standard treatment for advanced gastric cancer. ${ }^{7}$ In addition, new research is examining different modes of delivery (adjuvant, neoadjuvant, prophylactic HIPEC/systemic chemotherapy) and combinations of treatments (some of which have been discussed previously) to build on the promising results of CRS-HIPEC. These agents and approaches may be included in the future standard of care. Research is still ongoing to examine the effects of systemic chemotherapy in addition to HIPEC on gastric cancer survival. ${ }^{20}$ These include early postoperative chemotherapy (EPIC), ${ }^{53,54}$ and neoadjuvant intraperitoneal and systemic chemotherapy (NIPS). ${ }^{55} \mathrm{~A}$ number of randomized clinical trials are underway to evaluate both EPIC and NIPS in gastric cancer with PC. These chemotherapies are not without controversy. McConnell et al, ${ }^{53}$ provided data to show that EPIC plus HIPEC in combination increased complications to an unacceptable level and suggested that HIPEC-only treatment is preferred.

It is important to note that none of the current therapies for gastric cancers are strictly curative and that virtually 
all patients have subsequent recurrence and death. ${ }^{14}$ Still, HIPEC with CRS was found to have an $11 \%$ cure rate (no evidence of disease at 5 years) in patients that had PC in the setting of gastric cancer. ${ }^{56}$ Considering the high and rapid mortality associated with untreated disease gastric cancer with PC, CRS-HIPEC seems to be the best treatment currently available.

\section{The effect of HIPEC on gastric cancer morbidity}

In gastric cancer with PC, the rates of morbidity following CRS-HIPEC have been shown to be independent predictors of patient survival. ${ }^{10-35}$ These findings underscore the clinical value of avoiding significant morbidity in patients that have advanced gastric cancer. A particularly valuable clinical finding was described by Mizumoto et al, ${ }^{44}$ when they compared patients that received surgery alone with patients who received CRS-HIPEC, the CRS-HIPEC group had significantly reduced rates of postoperative morbidity.

One study by Tu et al looked at the safety and effectiveness of CRS-HIPEC. This study found low rates $(6.9 \%)$ of morbidity in response to the combined treatment regimen. ${ }^{35}$ Topgül et al, ${ }^{57}$ found that 8 of the 27 patients $(30 \%)$ in the study treated with CRS-HIPEC experienced perioperative morbidity, while one patient had HIPEC-related toxicity (3.9\%). In a powerful study by Desantis et al, ${ }^{38} 356$ patients underwent 401 consecutive CRS-HIPEC procedures with reported morbidity rates of $12.5 \%$ and a procedure mortality rate of $1 \%$. The study concludes that the $1 \%$ mortality rate and $12.5 \%$ morbidity rate are acceptably low when weighed against overall oncologic survival. ${ }^{37}$ Kang et al, ${ }^{31}$ compared gastrectomy alone against gastrectomy with HIPEC and showed that there was no significant difference in the rate of complication between the 2 groups. Wu et al, ${ }^{39}$ examined the complications associated with CRS-HIPEC and found that $15.2 \%$ of patients in their study had serious adverse events perioperatively with a $1.9 \%$ mortality rate. These authors also studied combined HIPEC and systemic chemotherapy as adjuvant therapy after CRS. They reported chemo toxicity morbidity rates (myelosuppression, nausea/vomiting) were $<10 \%$, an acceptably low rate considering the improved survival rates found in the study. ${ }^{22}$

One concern related to CRS-HIPEC is increased operative mortality. The mortality rates, however, have been shown to be low: $0 \%$ in a study by Yang et al, ${ }^{42} 0.9 \%$ in another study, ${ }^{35}$ and $3.5 \%$ in a larger study. ${ }^{43} \mathrm{~A} 30$-day mortality rate of $4.8 \%$ was found in a systematic review by Gill et al. ${ }^{58}$ Although the numbers vary, the consensus is 2-fold: The mortality risk is acceptably low for CRS-HIPEC candidates and careful patient selection, as mentioned previously, is key for reducing the risk of CRS-HIPEC associated morbidity.

A study by Polanco et al, ${ }^{59}$ set out to determine the learning curve for performing the CRS-HIPEC procedure in an academic medical center. They found that in order to achieve optimal outcomes (equal in terms of morbidity and mortality with experienced surgeons), 180 procedures were required to achieve the lowest risk of morbidity and greatest reduction in peritoneal tumor burden, with 90 procedures required to produce equal oncological survival outcomes. ${ }^{59}$ Sufficient training and experience may be a factor that influences the morbidity and mortality associated with CRS-HIPEC.

The chemotherapy approach to treatment has been studied in terms of timing relative to surgery to determine if there were advantages associated with certain regimens. In one study, patients with advanced gastric cancer were treated with either simultaneous CRS-HIPEC followed by systemic chemotherapy or neoadjuvant HIPEC with systemic chemotherapy and staged CRS followed by more rounds of systemic chemotherapy. While many of the metrics found these treatments to be comparable (no statistical difference), HIPEC with chemo and staged CRS showed better patient tolerance and compliance with no difference in overall survival. ${ }^{60}$ Thus, while CRS-HIPEC is generally well tolerated, there may be ways to make it even more tolerable. One study, conversely, by Hultman et al, ${ }^{61}$ found that overly aggressive combinations of therapy (in this case, several months of neoadjuvant chemotherapy followed by CRS-HIPEC plus early postoperative IPC) were associated with very high morbidity rates $(62.5 \%)$. The cohort of subjects in this study, however, was only 8 patients. ${ }^{61}$ Larger studies are needed to determine the relative efficacy of aggressive CRS-HIPEC regimens.

Differences in outcome based on differences in the CRS approach have also been the focus of studies. Berger et al, ${ }^{33}$ for example, showed that patients undergoing more extensive CRS-HIPEC ( $>5$ organs resected or $>3$ bowel anastomoses) had significantly increased rates of intraoperative blood loss, surgery duration, hospital stay, and 30-day morbidity (34\% vs $17.4 \%$ ). This finding is similar to one by Magge et al, ${ }^{37}$ which showed patients with $>2$ bowel anastomoses had poorer overall survival and disease progression. Another study by Franssen et $\mathrm{al}^{62}$ found that $\mathrm{PC}$ requiring diaphragmatic stripping or diaphragmatic resection was an independent predictor of 30-day morbidity (29\% vs $15 \%$ ). While Franssen et $\mathrm{al}^{62}$ did note increased morbidity in patients who received diaphragmatic stripping or resection, there was no difference in 90-day mortality rates. This finding suggests that the 
procedure is warranted in selected patients if it allows for improved cytoreduction.

A study by Yang et $\mathrm{al}^{63}$ focused primarily on the perioperative safety profile of CRS-HIPEC and followed some of the biomarkers for morbidity. The most common events they recorded were generalized edema and hypoproteinemia. Importantly, no patients included in this study had local or systemic infections, wound disruption, or other clinically important adverse events. Some of the more common intraand postoperative complications that were studied included longer duration of surgery, ${ }^{33}$ intraoperative blood loss, anastomotic leakage, surgical site infections and intra-abdominal abscesses, ${ }^{64}$ re-operation, longer hospital stay, respiratory complications, and chemotherapy or HIPEC-related toxicity. Overall, many of these studies either support CRS-HIPEC for reducing morbidity or find that the morbidity associated with CRS-HIPEC is acceptable given the associated increase in life expectancy.

HIPEC plays a role in palliating malignant ascites, a common and onerous morbidity associated with PC. In a retrospective study, Yuan et $\mathrm{al}^{16}$ showed that, when compared with traditional chemotherapy, HIPEC increases both survival and improves quality of life. The HIPEC population had decreased gastric cancer-related symptoms than those receiving chemotherapy alone, particularly the presence of malignant ascites was reduced. ${ }^{16}$ Similarly, in studies by Facchiano et al, ${ }^{65}$ and Yarema et al, ${ }^{25}$ they showed that, as well as providing survival benefit associated with CRS-HIPEC, HIPEC alone caused complete clinical regression of the symptoms of malignant ascites in patients with primary gastric cancer with PC. Remarkably, evidence for palliation of malignant ascites via HIPEC had already been described by the late 1990 s. ${ }^{66}$ For many patients with gastric cancer, the palliative benefit and reduction in cancer morbidity with the use of HIPEC is significant. This question of quality of life was also addressed by Tsilimparis et al, ${ }^{64}$ they found that patients undergoing CRS-HIPEC had quality of life after surgery similar to what it was preoperatively with most measures of quality of life recovering to normal levels within 6-12 months. They concluded that reduced quality of life was not an argument against denying these patients this beneficial treatment option.

In summary, while some specific questions remain regarding the safety of CRS-HIPEC, it seems to be reasonably well tolerated. Many cancer treatments prolong life at the cost of significant morbidity; however, there is evidence that CRSHIPEC may provide both morbidity and mortality benefit. This question of quality of life was also addressed by Tsil- imparis et al, ${ }^{64}$ they found patient quality of life after CRSHIPEC to be similar to what it was preoperatively. Moreover, most measures of quality of life recovered to normal levels within 6-12 months of undergoing CRS-HIPEC. In another study, Ji et al ${ }^{11}$ illustrated this principle by combining the data from published clinical trials on CRS-HIPEC. This study failed to find any statistically significant adverse effects that could be attributed to CRS-HIPEC. ${ }^{11}$ Thus, beyond being well tolerated, CRS-HIPEC may significantly decrease the incidence of some side effects, including malignant ascites.

\section{Translational research on HIPEC}

A number of studies have investigated HIPEC using transitional models. Graziosi et $\mathrm{al}^{67}$ showed that human gastric cancer cells that had been injected into mice responded well to HIPEC. Specifically, HIPEC reduced both the extent and severity of peritoneal dissemination of the cancer cells and increased the rates of survival in the HIPEC mouse group. On a molecular level, HIPEC was found to modulate the expression of genes that were important in the formation of peritoneal metastases. ${ }^{67}$ This work supports the approach of using HIPEC as a prophylactic means of preventing peritoneal metastases.

Some studies have evaluated HIPEC on rabbit models of gastric cancer. In one study, HIPEC was shown to increase overall survival by $70 \%$ in a rabbit model of gastric cancer with peritoneal metastases. ${ }^{68}$ While HIPEC was shown to benefit these rabbits significantly, PDOX, a prodrug peptide doxorubicin, was shown to further increase survival (beyond the benefit of HIPEC) by an additional $40 \%$. This study also showed that PDOX and HIPEC had a much more tolerable side-effect profile than the doxorubicin treatments. ${ }^{68}$ Similarly, in another study, Tang et $\mathrm{al}^{69}$ found that CRS-HIPEC could increase the survival of their diseased rabbits by $60 \%$. Moreover, based on peripheral blood cell counts, liver function, kidney function, and blood chemistries, the side effects were comparable between the control group and the CRSHIPEC group. Taken together, these studies demonstrate HIPEC to be both effective and relatively safe.

In vitro models of human cells have also been used to examine the effectiveness and safety of HIPEC. For example, one study analyzed long, non-coding RNA expression in human gastric cells. Long, non-coding RNA (LNCRNA) does not code for proteins and is thought to be involved in tumorigenesis. ${ }^{66}$ LNCRNAs were measured before and after exposure to HIPEC. The study showed that 154 LNCRNAs were up-regulated by more than double in response to HIPEC. ${ }^{70}$ Simultaneously, 412 LNCRNAs were downregulated by 
>2-fold. They hypothesize that LNCRNAs may underlie the treatment effectiveness of HIPEC. Zhang et al also studied the role of specific RNAs in human gastric cancer cells. Their work used miRNA microarrays to identify an 8-fold increase in miR-218 expression after CRS-HIPEC. ${ }^{71}$ Notably, miR-218 increased the sensitivity of the gastric cancer cells to in vitro cisplatin. miR-218. They concluded that miR-218 shows promise for reversing multidrug resistance in gastric cancer lines. These studies give molecular-level rationale to explain the efficacy of HIPEC while also highlighting molecular targets for future chemotherapeutic agents. The chemotherapy given by HIPEC may also have an effect on outcomes though there have been few studies done to compare one agent against another. Shetty et $\mathrm{al}^{72}$ showed that in MPM, HIPEC treatment with carboplatin resulted in significantly better outcomes than with the more traditional agent mitomycin $\mathrm{C}$. There is good evidence that the mitomycin $\mathrm{C}$ metabolizing enzyme NADPH Quinone Oxidoreductase 1 activity is reduced or absent in about $30 \%-40 \%$ of people due to polymorphism (C609T) in the active site. ${ }^{73}$ While not proven to be the cause of the poorer results with mitomycin in the MPM patients, the presence of these polymorphisms should be taken into consideration when selecting the chemotherapy agent for HIPEC treatment of peritoneal metastasis from gastric and other concerns.

A number of tumor markers have been investigated in gastric cancer to help determine prognosis and the effectiveness of therapy. The 3 most common markers are carcinoembryonic antigen (CEA), CA 19-0, and CA 72.4. Of these, CEA has received the most attention. CEA is most widely used for colon cancer as a prognostic indicator and mediator of hepatic metastasis. ${ }^{74}$ In gastric cancer, all 3 markers have been associated with both stage and survival. ${ }^{75}$ While no prospective trials have been carried out, studies have indicated that measurement of serum levels of these markers in combination, can be used for preoperative staging and monitoring the effects of therapy postoperatively and after chemotherapy. ${ }^{71}$ Additionally, Wang et al, ${ }^{76}$ reported that an evaluation of CEA tissue staining showed good correlations with serum CEA and prognosis. Jung et al measured CEA in both serum and ascites from 119 patients with peritoneal metastasis. This study showed median CEA levels of $130.5 \mathrm{ng} / \mathrm{mL}$ in ascites with much lower serum CEA levels (Median $2.1 \mathrm{ng} / \mathrm{mL}){ }^{76}$ Patients with low ascites CEA $(<5$ $\mathrm{ng} / \mathrm{mL}$ ) had a significantly longer survival ( 7.4 months) than those with higher CEA levels (2.3 months). ${ }^{77}$ Asao et $\mathrm{al}^{78}$ examined peritoneal washings from gastric cancer patients and determined that CEA could be an important indicator of peritoneal dissemination in the absence of visible tumor. Ito et $\mathrm{al}^{79}$ also looked at peritoneal washings by detecting CEA mRNA as an indicator of the presence of tumor cells and came up with similar conclusions. These studies indicate that there is a potential role for tumor marker measurements in gastric cancer with $\mathrm{PC}$ and measurement of CEA in pre and post CRS-HIPEC patients may be a significant indicator of future outcomes.

A study by Coccolini et $\mathrm{al}^{80}$ evaluated variations in cytokines during CRS-HIPEC in patients with a variety of tumor types (including gastric cancer), all of which had peritoneal metastases. Cancer, surgery, chemotherapy and hyperthermia are stressful factors that induce the systemic inflammatory response system that has been best studied in conjunction with sepsis and septic shock. Inflammatory markers studied, that are commonly measured in this setting, included tumor necrosis factor- $\alpha$ (TNF- $\alpha$ ), interleukin-6 (IL-6, CA-125, and procalcitonin (PCT). Coccolini et $\mathrm{al}^{80}$ showed a steady state of TNF- $\alpha$ and CA-125 throughout the procedure and sharp, significant increases in PCT and IL-6 during the hyperthermic portion of the HIPEC infusion. Changes in inflammatory cytokines in response to CRS-HIPEC mimic those of septic shock and are probably due to hemodynamic and temperature instability from HIPEC infusion and surgery. Management of the hemodynamic status and temperature of the patient by the anesthesiologist during surgery is crucial to the procedure's success. They proposed that HIPEC could be a good experimental model for testing different anesthetic approaches to see if they can have a positive effect on the inflammatory response to surgery and hyperthermia.

\section{Other recent research on HIPEC in the setting of gastric cancer and/or peritoneal metastases}

Given that gastric cancer with PC has such a poor survival, even with promising results from CRS-HIPEC, there has been discussion of the potential role for intraperitoneal immunotherapy for treating gastric cancer. Currently, Goéré et al ${ }^{81}$ are conducting a Phase II clinical trial on gastric cancer patients with PC using an intraperitoneal infusion of catumaxomab following CRS with the hopes of showing improved outcomes over CRS-HIPEC. Catumaxomab has the ability to bind to cells expressing epithelial cell adhesion molecule, which is expressed by up to $90 \%$ of gastric carcinomas. ${ }^{81}$ An earlier study by Stroehlein et $\mathrm{al}^{82}$ did a small trial of 9 patients with PC from different primary cancers (including 6 with gastric cancer) to test the use of intraperitoneal infusion of trifunctional antibodies. The purpose of the immunotherapy 
was to redirect cytotoxic CD8+ T lymphocytes to tumor cells and induce active tumor immunity. The study showed that in 5/9 patients, tumor reactive $T$ lymphocytes increased significantly, indicating specific antitumor immunity. They concluded that immunotherapy using trifunctional antibodies may provide a new option for treatment of PC and should be evaluated in ongoing clinical trials. Animal models have shown that it is possible to shrink and even eliminate PC using intraperitoneal immunotherapy. ${ }^{83} \mathrm{Fu}$ et al compared HIPEC with combined HIPEC and intraperitoneal immunotherapy with IL-2 to stimulate the immune system. They found that intraperitoneal IL-2 immunotherapy significantly increased Th1 cytokines to induce tumor killing and increased the 3 -year survival of the patients by $18.1 \%$ compared with HIPEC alone. ${ }^{84}$ They also showed that in the HIPEC plus immunotherapy group, the recurrence and liver metastasis rates were significantly lower and concluded that immunotherapy has potential for treatment of PC and prevention of recurrence and metastases.$^{84}$ Very few studies have been done in this arena, but there is growing interest in the potential for immunotherapy treatments to improve the outcomes of patients with gastric cancer or cancers with peritoneal metastases.

\section{Conclusion}

Gastric cancer continues to be a significant disease in the international community with poor survival rates and few treatment options. Gastric cancer, as well as a variety of other primary cancers, often metastasize to the peritoneum. Unfortunately, cancer with peritoneal metastases represents a particularly grim subset of disease ${ }^{85} \mathrm{CRS}$ with HIPEC has been shown to be an effective treatment regimen in a variety of cancers with PC, most especially in PMP for which it is the standard of care. The data for gastric cancer, however, are not as conclusive and many studies have been done on the morbidity and mortality of this procedure. Additionally, variations of the CRS-HIPEC regimen have been investigated in an ongoing search for better treatment outcomes. Different modes of delivery (adjuvant, neoadjuvant, prophylactic HIPEC/systemic chemotherapy) and combinations of treatments have been shown to build on the promising results of CRS-HIPEC and may be included in standards of care in the future.

CRS-HIPEC has been shown to be superior to either CRS alone or systemic chemotherapy alone in terms of improving survival. Consequently, CRS-HIPEC seems to be the best current treatment for patients with gastric cancer and peritoneal metastases. For this reason, the international community has recommended it as the treatment for this disease. Importantly, many studies suggest the use of prudent patient selection before CRS-HIPEC as many subgroups of patients have been shown to uniquely benefit from the treatment, while others have not. Prudent patient selection will allow clinicians to identify those that are most likely to enjoy significant mortality benefits from CRS-HIPEC.

Studies on CRS-HIPEC have shown the procedure to have acceptably low rates of morbidity and peri- and postoperative complications as well as significant reductions in the incidence of malignant ascites associated with PC.

Ongoing translational research on HIPEC supports the use of HIPEC as prophylaxis for prevention of peritoneal metastasis, and demonstrates HIPEC to be both effective and safe in animal models. In addition, measurement of the tumor marker CEA has been shown to be an effective indicator of future outcomes in gastric cancer treated with CRS-HIPEC. Although the treatment outcomes have improved, even with current treatment using CRS-HIPEC for gastric cancer with PC, the survival rates can be dismal. The treatment of advanced gastric cancer with $\mathrm{PC}$ is an ongoing field of study and further work on neoadjuvant chemotherapy (EPIC and NIPS) is needed to supplement HIPEC. Other studies are also underway to examine the effectiveness of catumaxumab. This drug is given intraperitoneally and shows promise for the treatment of malignant ascites ${ }^{86,87}$ Future directions for the treatment of gastric cancer with PC are likely to include the use of intraperitoneal immunotherapy.

\section{Disclosure}

The authors report no conflicts of interest in this work.

\section{References}

1. Ferlay J, Soerjomataram I, Bray F, et al. Cancer incidence and mortality worldwide: sources, methods and major patterns in GLOBOCAN 2012. Int J Cancer. 2015;136(5):E359-E386.

2. Van Leeuwen B, Graf W, Pahlman L, Mahteme H. Swedish experience with peritonectomy and HIPEC. HIPEC in peritoneal carcinomatosis. Ann Surg Oncol. 2008;15(3):745-753.

3. Riihimäki M, Hemminki A, Sundquist K, Sundquist J, Hemminki K. Metastatic spread in patients with gastric cancer. Oncotarget 2016;7(32):52307-52316.

4. American Cancer Society [webpage on the Internet]. Survival rates for stomach cancer by age; 2014. Available from: https://www.cancer.org/ cancer/stomach-cancer/detection-diagnosis-staging/survival-rates.html. Accessed April 19, 2017.

5. Yang D, Hendifar A, Lenz C, et al. Survival of metastatic gastric cancer: significance of age, sex and race/ethnicity. $J$ Gastroint Oncol. 2011;2(2):77-84.

6. Kodera Y. Gastric cancer with minimal peritoneal metastasis: is this a sign to give up or to treat more aggressively? Nagoya J Med Sci.2013;75(1-2): 3-10.

7. Li Y, Zhou Y, Yang G, et al. Chinese expert consensus on cytoreductive surgery and hyperthermic intraperitoneal chemotherapy for peritoneal malignancies. World J Gastroenterol. 2016;22(30):6906-6916. 
8. Elias D, Goéré D, Ducreux M, et al. Role of hyperthermic intraoperative peritoneal chemotherapy in the management of peritoneal metastases. Eur J Cancer. 1990;50(2):332-340.

9. Ni Z, Li C, Zhu Z, et al. Efficacy and safety of surgery combined with hyperthermic intraperitoneal chemotherapy in the treatment of advanced gastric cancer: a meta-analysis. Zhonghua Wei Chang Wai Ke Za Zhi. 2016;19(12):1406-1413.

10. Zeng W, Hu W, Li Y, et al. Long term follow up and retrospective study on 533 gastric cancer cases. BMC Surg. 2014;14(1):29-37.

11. Ji Z, Peng K, Li Y, et al. Current status and future prospects of clinical trials on CRS + HIPEC for gastric cancer peritoneal metastases. Int $J$ Hyperthermia. 2017;33(5):562-570.

12. Yang X, Huang C, Li Y, et al. Cytoreductive surgery and hyperthermic intraperitoneal chemotherapy improves survival of patients with peritoneal carcinomatosis from gastric cancer: final results of a phase III randomized clinical trial. Ann Surg Oncol. 2011;18(6);1575-1581.

13. Coccolini F, Cotte E, Ansaloni L, et al. Intraperitoneal chemotherapy in advanced gastric cancer. Meta-analysis of randomized trials. Eur $J$ Surg Oncol. 2014;40(1);12-26.

14. Boerner T, Graichen A, Jeiter T, et al. CRS-HIPEC prolongs survival but is not curative for patients with peritoneal carcinomatosis of gastric cancer. Ann Surg Oncol. 2016;23(12):3972-3977.

15. Wu X, Yuan P, Ji J, et al. Cytoreductive surgery and hyperthermic intraperitoneal chemotherapy improves the survival of gastric cancer patients with ovarian metastasis and peritoneal dissemination. Tum Biol. 2013;34(1):463-469.

16. Yuan M, Wang Z, Zhong H, et al. A retrospective analysis of hyperthermic intraperitoneal chemotherapy for gastric cancer with peritoneal metastasis. Mol Clin Oncol. 2016;5(2):395-399.

17. Rudloff U, Langan R, Avital I, et al. Impact of maximal cytoreductive surgery plus regional heated intraperitoneal chemotherapy (HIPEC) on outcome of patients with peritoneal carcinomatosis of gastric origin: results of the GYMSSA trial. $J$ Surg Oncol. 2014;110(3): 275-284.

18. Zhibing W, Qinghua D, Ruzhen Z, et al. Clinical study of cisplatin hyperthermic intraperitoneal perfusion chemotherapy in combination with docetaxel, 5-flourouracil and leucovorin intravenous chemotherapy for the treatment of advanced-stage gastric carcinoma. Hepatogastroenterology. 2013;60(125):989-994.

19. Yonemura Y, Ishibashi H, Li Y, et al. Effects of neoadjuvant laparoscopic hyperthermic intraperitoneal chemotherapy and neoadjuvant intraperitoneal/systemic chemotherapy on peritoneal metastases from gastric cancer. Ann Surg Oncol. 2017;24(2):478-485.

20. Glehen O, Gilly F, Elias D, et al. Peritoneal carcinomatosis from gastric cancer: a multi-institutional study of 159 patients treated by cytoreductive surgery combined with perioperative intraperitoneal chemotherapy. Ann Surg Oncol. 2010;17(9):2370-2377.

21. Schildberg C, Weidinger T, Boxberger F, et al. Metastatic adenocarcinomas of the stomach or esophagogastric junction (UICC stage IV) are not always a palliative situation: a retrospective analysis. World J Surg. 2014;38(2):419-425.

22. Wu Z, Ma S, Li X, et al. Effect of hyperthermic intraperitoneal perfusion chemotherapy in combination with intravenous chemotherapy as postoperative adjuvant therapy for advanced gastric cancer. Hepatogastroenterology. 2014;61(132):972-977.

23. Canbay E, Mizumoto A, Yonemura Y, et al. Outcome data of patients with peritoneal carcinomatosis from gastric origin treated by a strategy of bidirectional chemotherapy prior to cytoreductive surgery and hyperthermic intraperitoneal chemotherapy in a single specialized center in Japan. Ann Surg Oncol. 2014;21(4):1147-1152.

24. Saladino E, Fleres F, Macrì A, et al. The role of prophylactic hyperthermic intraperitoneal chemotherapy in the management of serosal involved gastric cancer. Anticancer Res. 2014;34(4):2019-2022.

25. Yarema R, Ohorchak M, Fetsych T, et al. Hyperthermic intraperitoneal chemoperfusion in combined treatment of locally advanced and disseminated gastric cancer: results of a single-center retrospective study. Int J Hyperthermia. 2014;30(3):159-165.
26. Costa W, Coimbra F, Mello C, et al. Safety and preliminary results of perioperative chemotherapy and hyperthermic intraperitoneal chemotherapy (HIPEC) for high-risk gastric cancer patients. World J Surg Oncol. 2012;10:195.

27. Li C, Yan M, Lin Y, et al. Surgical resection with hyperthermic intraperitoneal chemotherapy for gastric cancer patients with peritoneal dissemination. J Surg Oncol. 2010;102(5):361-365.

28. Geng X, Liu H, Li G, et al. Survival benefit of gastrectomy for gastric cancer with peritoneal carcinomatosis: a propensity score-matched analysis. Cancer Med. 2016;5(10):2781-2791.

29. Graziosi L, Cantarella F, Donini A, et al. Preliminary results of prophylactic HIPEC in patients with locally advanced gastric cancer. Ann Ital Chir. 2013;84(5):551-556.

30. Coccolini F, Celotti A, Ansaloni L, et al. Hyperthermic intraperitoneal chemotherapy (HIPEC) and neoadjuvant chemotherapy as prophylaxis of peritoneal carcinomatosis from advanced gastric cancer-effects on overall and disease free survival. J Gastrointest Oncol. 2016;7(4):523-529.

31. Kang L, Mok K, Chou N, et al. Intraoperative hyperthermic intraperitoneal chemotherapy as adjuvant chemotherapy for advanced gastric cancer patients with serosal invasion. J Chin Med Assoc. 2013;76(8):425-431.

32. Halkia E, Kopanakis N, Spiliotis J, et al. Is cholecystectomy and removal of the round ligament of the liver a necessary step in cytoreductive surgery and HIPEC, for peritoneal carcinomatosis?. Ann Ital Chir. 2015;86(4):323-326.

33. Berger Y, Aycart S, Mandeli J, Heskel M, Sarpel U, Labow D. Extreme cytoreductive surgery and hyperthermic intraperitoneal chemotherapy: outcomes from a single tertiary center. Surg Oncol. 2015;24(3):264-269.

34. American Cancer Society [webpage on the Internet]. What are the key statistics about stomach cancer?; 2014. Available from: https://www. cancer.org/cancer/stomach-cancer/about/key-statistics.html. Accessed April 19, 2017.

35. Hall JJ, Loggie BW, Shen P, et al. Cytoreductive surgery with intraperitoneal hyperthermic chemotherapy for advanced gastric cancer. $J$ Gastrointest Surg. 2004;8(4):454-463.

36. Tu Y, Tian Y, Cui S, et al. Cytoreductive surgery combined with hyperthermic intraperitoneal chemoperfusion for the treatment of gastric cancer: a single-center retrospective study. Int J. 2016;32(6):587-594.

37. Magge D, Zenati M, Choudry H, et al. Aggressive loco regional surgical therapy for gastric peritoneal carcinomatosis. Ann Surg Oncol. 2014;21(5):1448-1455.

38. Desantis M, Bernard J, Bereder J, et al. Morbidity, mortality, and oncological outcomes of 401 consecutive cytoreductive procedures with hyperthermic intraperitoneal chemotherapy (HIPEC). Langenbeck's Arch Surg. 2015;400(1):37-48.

39. Wu H, Peng K, Li Y, et al. Cytoreductive surgery plus hyperthermic intraperitoneal chemotherapy with lobaplatin and docetaxel to treat synchronous peritoneal carcinomatosis from gastric cancer: results from a Chinese center. Eur J Surg Oncol. 2016;42(7):1024-1034.

40. Graziosi L, Marino E, Donini A. Role of CRS plus HIPEC in gastric cancer peritoneal carcinomatosis. J Surg Oncol. 2015;111(2):248.

41. Königsrainer I, Horvath P, Struller F, Königsrainer A, Beckert S. Initial clinical experience with cytoreductive surgery and hyperthermic intraperitoneal chemotherapy in signet-ring cell gastric cancer with peritoneal metastases. J Gastric Cancer. 2014;14(2):117-122.

42. Yang X, Li Y, Yonemura Y. Cytoreductive surgery plus hyperthermic intraperitoneal chemotherapy to treat gastric cancer with ascites and/or peritoneal carcinomatosis: results from a Chinese center. J Surg Oncol. 2010;101(6):457-464.

43. Müller H, Hotopp T, Tofeili A, Wutke K. Systemic chemotherapy using FLOT - regimen combined with cytoreductive surgery plus HIPEC for treatment of peritoneal metastasized gastric cancer. Hepatogastroenterology. 2014;61(131):703-706.

44. Mizumoto A, Canbay E, Yonemura Y, et al. Morbidity and mortality outcomes of cytoreductive surgery and hyperthermic intraperitoneal chemotherapy at a single institution in Japan. Gastroenterol Res Pract. 2012;83:642-645. 
45. Bhagwandin S, Naffouje S, Salti G. Utility of chemoresponse assay in patients undergoing cytoreductive surgery plus hyperthermic intraperitoneal chemotherapy. Ann Surg Oncol. 2015;22(8):2573-2577.

46. Macrì A, Saladino E, Famulari C, et al. Cytoreductive surgery plus hyperthermic intraperitoneal chemotherapy in elderly patients. In Vivo. 2011;25(4):687-690

47. Kim K, Chow O, Hiotis S, et al. Peritoneal carcinomatosis in patients with gastric cancer, and the role for surgical resection, cytoreductive surgery, and hyperthermic intraperitoneal chemotherapy. Am J Surg 2014;207(1):78-83.

48. Votanopoulos K, Newman N, Levine E, et al. Outcomes of cytoreductive surgery (CRS) with hyperthermic intraperitoneal chemotherapy (HIPEC) in patients older than 70 years; survival benefit at considerable morbidity and mortality. Ann Surg Oncol. 2013;20(11):3497-3503.

49. Elias D, Gilly F, Glehen O, et al. Pseudomyxoma peritonei: a French multicentric study of 301 patients treated with cytoreductive surgery and intraperitoneal chemotherapy. Eur J Surg Oncol. 2010;36(5):456-462.

50. Wu X, Yuan P, Ji J, et al. Cytoreductive surgery and hyperthermic intraperitoneal chemotherapy improves the survival of gastric cancer patients with ovarian metastasis and peritoneal dissemination. Tum Biol. 2013;34(1):463-469.

51. Seshadri R, Glehen O. The role of hyperthermic intraperitoneal chemotherapy in gastric cancer. Indian J Surg Oncol. 2016;7(2):198-207.

52. Scaringi S, Kianmanesh R, Msika S, et al. Advanced gastric cancer with or without peritoneal carcinomatosis treated with hyperthermic intraperitoneal chemotherapy: a single western center experience. Eur J Surg Oncol. 2008;34(11):1246-1252.

53. McConnell YJ, Mack LA, Francis WP, Ho T, Temple WJ. HIPEC+ EPIC versus HIPEC-alone: differences in major complications following cytoreduction surgery for peritoneal malignancy. J Surg Oncol. 2013;107(6):591-596.

54. Goodman MD, McPartland S, Detelich D, Saif MW. Chemotherapy for intraperitoneal use: a review of hyperthermic intraperitoneal chemotherapy and early post-operative intraperitoneal chemotherapy. $J$ Gastrointest Oncol. 2016;7(1):45-57.

55. Montori G, Coccolini F, Ceresoli M, et al. The treatment of peritoneal carcinomatosis in advanced gastric cancer: state of the art. Int J Surg Oncol. 2014;(2014), Article ID 912418.

56. Chia C, You B, Glehen O, et al. Patients with peritoneal carcinomatosis from gastric cancer treated with cytoreductive surgery and hyperthermic intraperitoneal chemotherapy: is cure a possibility? Ann Surg Oncol. 2016;23(6):1971-1979.

57. Topgül K, Çetinkaya M, Malazgirt Z, et al. Cytoreductive surgery (SRC) and hyperthermic intraperitoneal chemotherapy (HIPEC) for treatment of peritoneal carcinomatosis: our initial experience and technical details. Ulus Cerrahi Derg. 2015;31(3):138-147.

58. Gill R, Al-Adra D, Schiller D, et al. Treatment of gastric cancer with peritoneal carcinomatosis by cytoreductive surgery and HIPEC: a systematic review of survival, mortality, and morbidity. J Surg Oncol. 2011;104(6):692-698.

59. Polanco P, Ding Y, Choudry H, et al. Institutional learning curve of cytoreductive surgery and hyperthermic intraperitoneal chemoperfusion for peritoneal malignancies. Ann Surg Oncol. 2015;22(5):1673-1679.

60. Wu X, Li Z, Ji J, et al. Hyperthermic intraperitoneal chemotherapy plus simultaneous versus staged cytoreductive surgery for gastric cancer with occult peritoneal metastasis. J Surg Oncol. 2015;111(7):840-847.

61. Hultman B, Lind P, Mahteme H, et al. Phase II study of patients with peritoneal carcinomatosis from gastric cancer treated with preoperative systemic chemotherapy followed by peritonectomy and intraperitoneal chemotherapy. Acta Oncol. 2013;52(4):824-830.

62. Franssen B, Tabrizian P, Sarpel U, et al. Outcome of cytoreductive surgery and hyperthermic intraperitoneal chemotherapy on patients with diaphragmatic involvement. Ann Surg Oncol. 2015;22(5):1639-1644.

63. Yang X, Li Y, Yonemura Y, et al. Cytoreductive surgery plus hyperthermic intraperitoneal chemotherapy improves survival in selected patients with peritoneal carcinomatosis from abdominal and pelvic malignancies: results of 21 cases. Ann Surg Oncol. 2009;16(2):345-351.
64. Tsilimparis N, Bockelmann C, Hartmann J, et al. Quality of life in patients after cytoreductive surgery and hyperthermic intraperitoneal chemotherapy: is it worth the risk? Ann Surg Oncol. 2013;20(1): 226-232.

65. Facchiano E, Scaringi S, Msika S, et al. Laparoscopic hyperthermic intraperitoneal chemotherapy (HIPEC) for the treatment of malignant ascites secondary to unresectable peritoneal carcinomatosis from advanced gastric cancer. Eur J Surg Oncol. 2008;34(2):154-158.

66. Loggie B, Perini M, Fleming R, Russell G, Geisinger K. Treatment and prevention of malignant ascites associated with disseminated intraperitoneal malignancies by aggressive combined-modality therapy. Am Surg. 1997;63(2):137-143.

67. Graziosi L, Mencarelli A, Fiorucci S, et al. Gene expression changes induced by HIPEC in a murine model of gastric cancer. In Vivo. 2012;26(1):39-45.

68. Tang L, Duan R, Li Y, et al. Synthesis, identification and in vivo studies of tumor-targeting agent peptide doxorubicin (PDOX) to treat peritoneal carcinomatosis of gastric cancer with similar efficacy but reduced toxicity. Mol Cancer. 2014;13(1):44-61.

69. Tang L, Mei L, Li Y, et al. Cytoreductive surgery plus hyperthermic intraperitoneal chemotherapy improves survival of gastric cancer with peritoneal carcinomatosis: evidence from an experimental study. JTransl Med. 2011;9(1):53.

70. Zeng X, Shi H, Wang J, Cui S, Tang H, Zhang X. Long noncoding RNA aberrant expression profiles after cytoreductive surgery and hyperthermic intraperitoneal chemotherapy of AGC ascertained by microarray analysis. Tum Biol. 2015;36(7):5021-5029.

71. Zhang X, Shi H, Wang L, et al. MicroRNA-218 is upregulated in gastric cancer after cytoreductive surgery and hyperthermic intraperitoneal chemotherapy and increases chemosensitivity to cisplatin. World $J$ Gastroenterol. 2014;20(32):11347-11355.

72. Shetty S, Bathia L, Govindarajan V, Thomas P, Loggie B. Comparison of visceral sparing cytoreductive surgery and hyperthermic intraperitoneal chemotherapy with mitomycin or carboplatin for diffuse malignant peritoneal mesothelioma. Am Surg. 2014;80(4):348-352.

73. Fleming RA, Drees J, Loggie BW, et al. Clinical significance of a NAD (P) H: quinone oxidoreductase 1 polymorphism in patients with disseminated peritoneal cancer receiving intraperitoneal hyperthermic chemotherapy with mitomycin C. Pharmacogenetics. 2002;12(1): 31-37.

74. Thomas P, Forse R, Bajenova O. Carcinoembryonic antigen (CEA) and its receptor hnRNP M are mediators of metastasis and the inflammatory response in the liver. Clin Exp Metastasis. 2011;28(8):923-932.

75. Shimada H, Noie T, Ohashi M, Oba K, TakahashiY. Clinical significance of serum tumor markers for gastric cancer: a systematic review of literature by the Task Force of the Japanese Gastric Cancer Association. Gastric Cancer. 2014;17(1):26-33.

76. Wang W, Seeruttun S, Zhou Z, et al. Prognostic significance of carcinoembryonic antigen staining in cancer tissues of gastric cancer patients. Ann Surg Oncol. 2016;23(4):1244-1251.

77. Jung M, Jeung HC, Lee SS, et al. The clinical significance of ascitic fluid CEA in advanced gastric cancer with ascites. J Cancer Res Clin Oncol. 2010;136(4):517-526.

78. Asao T, Fukuda T, Yazawa S, Nagamachi Y. Carcinoembryonic antigen levels in peritoneal washings can predict peritoneal recurrence after curative resection of gastric cancer. Cancer. 1991;68(1):44-47.

79. Ito S, Nakanishi H, Kodera Y, Mochizuki Y, Tatematsu M, Yamamura Y. Prospective validation of quantitative CEA mRNA detection in peritoneal washes in gastric carcinoma patients. $\mathrm{Br} J$ Cancer. 2005;93(9):986-992.

80. Coccolini F, Corbella D, Ansaloni L, et al. Time course of cytokines, hemodynamic and metabolic parameters during hyperthermic intraperitoneal chemotherapy. Minerva Anestesiol. 2016;82(3):310-319.

81. Goéré D, Gras-Chaput N, Elias D, et al. Treatment of gastric peritoneal carcinomatosis by combining complete surgical resection of lesions and intraperitoneal immunotherapy using catumaxomab. BMC Cancer. 2014;14(1):148-154. 
82. Stroehlein M, Jaeger M, Lindhofer H, Peschel C, Jauch K, Heiss M. Intraperitoneal immunotherapy of peritoneal carcinomatosis from solid tumors by trifunctional antibodies. J Clin Oncol. 2004;22(14): 2532.

83. Piso P, Aselmann H, von Wasielewski R, Dahlke M, Klempnauer J, Schlitt H. Prevention of peritoneal carcinomatosis from human gastric cancer cells by adjuvant-type intraperitoneal immunotherapy in a SCID mouse model. Eur Surg Res. 2003;35(6):470-476.

84. Fu Q, Meng F, Shen X, Guo R. Efficacy of intraperitoneal thermochemotherapy and immunotherapy in intraperitoneal recurrence after gastrointestinal cancer resection. World J Gastroenterol. 2002;8(6): 1019-1022.
85. Franko J, Shi Q, Goldman CD, et al. Treatment of colorectal peritoneal carcinomatosis with systemic chemotherapy: a pooled analysis of north central cancer treatment group phase III trials N9741 and N9841. J Clin Oncol. 2012;30(3):263-267.

86. Heiss MM, Murawa P, Koralewski P, et al. The trifunctional antibody catumaxomab for the treatment of malignant ascites due to epithelial cancer: results of a prospective randomized phase II/III trial. Int $J$ Cancer. 2010;127(9):2209-2221.

87. Ott MG, Marmé F, Moldenhauer G, et al. Humoral response to catumaxomab correlates with clinical outcome: results of the pivotal phase II/III study in patients with malignant ascites. Int $J$ Cancer. 2012;130(9):2195-2203.
Gastrointestinal Cancer: Targets and Therapy

\section{Publish your work in this journal}

Gastrointestinal Cancer: Targets and Therapy is an international, peer-reviewed, open access journal focusing on gastro-intestinal cancer research, identification of therapeutic targets and the optimal use of preventative and integrated treatment interventions to achieve improved outcomes, enhanced survival and quality of life for the
Dovepress

cancer patient. The manuscript management system is completely online and includes a very quick and fair peer-review system. Visit http://www.dovepress.com/testimonials.php to read real quotes from published authors.

Submit your manuscript here: https://www.dovepress.com/gastro-intestinal-cancer-targets-and-therapy-journal 